\title{
Fair Pairwise Exchange among Groups
}

\author{
Zhaohong Sun ${ }^{1}$, Taiki Todo ${ }^{2}$ and Toby Walsh ${ }^{1}$ \\ ${ }^{1}$ UNSW Sydney \\ ${ }^{2}$ Kyushu University \\ \{zhaohong.sun, t.walsh\}@unsw.edu.au, todo@inf.kyushu-u.ac.jp
}

\begin{abstract}
We study the pairwise organ exchange problem among groups motivated by real-world applications and consider two types of group formulations. Each group represents either a certain type of patientdonor pairs who are compatible with the same set of organs, or a set of patient-donor pairs who reside in the same region. We address a natural research question, which asks how to match a maximum number of pairwise compatible patient-donor pairs in a fair and individually rational way. We first propose a natural fairness concept that is applicable to both types of group formulations and design a polynomial-time algorithm that checks whether a matching exists that satisfies optimality, individual rationality, and fairness. We also present several running time upper bounds for computing such matchings for different graph structures.
\end{abstract}

\section{Introduction}

Due to a shortage of organs harvested from deceased donors, living donations have become a significant approach to saving the lives of patients who suffer from serious organ dysfunction. One issue with living donations is that the organs from willing donors may be medically incompatible with the intended patients. This problem can be partially overcome by organ exchange, which allows patients to swap their donors with others to obtain compatible organs [Roth et al., 2004]. Since transplant operations are usually conducted simultaneously, a fixed upper bound is always imposed on the length of the exchange cycles. Pairwise organ exchange, which is the most common form in real-life, involves two pairs of patient and donor [Roth et al., 2005]. The design of organ exchange market has attracted considerable attention from both the economics and computer science fields [Abraham et al., 2007; Bertsimas et al., 2013; Dickerson et al., 2014; Hajaj et al., 2015; Dickerson and Sandholm, 2017; Dickerson et al., 2019; Ergin et al., 2020; Freedman et al., 2020].

In this paper, we study the organ exchange problem from a different perspective. We concentrate on a pairwise organ exchange market where all the patient-donor pairs are partitioned into disjoint groups. Dividing patient-donor pairs into groups is motivated by applications. Next we describe two types of group formulations that have surfaced in recent literature.

In the first type, a group of agents represents a certain type of patient-donor pairs where patients are compatible with the same set of organs in the market. For instance, Dickerson et al. [2017] introduced a new model for kidney exchange that classifies all participating patient-donor pairs into a fixed number of types, based on a common set of attributes, e.g., blood type, tissue type, age, insurance, willingness to travel, etc. Ergin et al. [2017] studied the dual-donor organ exchange problem w.r.t. lung and liver exchanges. They consider a simplified model for theoretical analysis and grouped patientdonor pairs by blood types without taking tissue type or size compatibility into account.

In the second type, each group represents a set of patientdonor pairs located in a certain area, formed geographically. For instance, each group can represent a set of patient-donor pairs in a certain hospital [Ashlagi et al., 2015]. Each group can also represent a state or territory in the national kidney exchange market, e.g. the Australian Organ and Tissue Authority [Mattei et al., 2017]. Or each group can represent a particular country in the European organ exchange program [Biró et al., 2019].

Although dividing patient-donors into groups was previously proposed in the literature, scant attention was paid to the fair allocation of patient-donor pairs among groups. One exception [Biró et al., 2019] considered core allocations, i.e., outcomes that cannot be improved upon by a coalition of agents. However, the core may be empty, and it is co-NPhard to check its existence. In contrast, we focus on the following research question: how can we design efficient algorithms that match a maximum number of pairwise compatible patient-donor pairs in a fair and individual rational way?

The contributions of this paper are summarized as follows. First, we propose a straightforward fairness concept for organ exchange among groups based on the notion of selection ratio, which provides the flexibility to capture different ideas such as egalitarianism and proportionality. Second, we introduce a general polynomial-time algorithm that finds a matching that satisfies maximality, individual rationality, and fairness whenever it exists. Third, we provide several running time upper bounds of computing such matchings for different graph structures w.r.t. two forms of group formulations. 


\section{Model}

We consider a pairwise organ exchange problem by compatibility graph $G=(V, E)$ where each vertex $v \in V$ represents a patient-donor pair. We assume that for each vertex, the patient is incompatible with his donor, or they will conduct the transplant immediately rather than participating in the exchange program [Roth et al., 2007].

Edge set $E$ is specified as follows. There is an edge between two vertices, $i, j \in V$, if the donor of pair $i$ is compatible with the patient of pair $j$ and the donor of pair $j$ is compatible with the patient of pair $i$. Vertex set $V$ is partitioned into $k$ disjoint sets where each set $V_{i} \subseteq V$ represents a group of patient-donor pairs. Let $P=\left\{V_{1}, \cdots, V_{k}\right\}$ denote the partition of vertices, i.e., $V_{i} \cap V_{j}=\emptyset$ for any $i \neq j$ and $\bigcup_{V_{i} \in P} V_{i}=V$.

Depending on whether all vertices within the same group have the same set of neighbors, we consider two types of compatible graph structures that correspond to two types of group formulations. If all the vertices within the same group have the same set of neighbors, then we refer to such graphs as compatible graphs with identical neighbors.

A matching $M$ in $G$ is a set of edges without common vertices, and a maximum matching is a matching that contains the largest possible number of edges. Let $\mathbb{M}$ denote the set of all possible matchings in $G$ and let $\mathbb{M}^{*}$ denote the set of all maximum matchings in $G$. Given a matching $M$ in $G$, let $\left|M_{i}\right|$ denote the number of matched vertices from group $V_{i}$. For each group $V_{i}$, let $\max \left(V_{i}\right)=\max _{M \in \mathbb{M}^{*}}\left|M_{i}\right|$ denote the maximum bound of matched vertices from group $V_{i}$ among $\mathbb{M}^{*}$, and let $\min \left(V_{i}\right)=\min _{M \in \mathbb{M}^{*}}\left|M_{i}\right|$ denote the minimum bound of matched vertices from group $V_{i}$ among $\mathbb{M}^{*}$.

Let $G\left[V_{i}\right]$ denote a subgraph of $G$ only induced by vertices from group $V_{i}$. Consider any maximum matching $M^{\prime}$ in subgraph $G\left[V_{i}\right]$. Let $\widetilde{\min }\left(V_{i}\right)=2 \cdot\left|M^{\prime}\right|$ denote the modified minimum bound of group $V_{i}$, which equals twice the size of maximum matching $M^{\prime}$ in $G\left[V_{i}\right]$. Note that the modified minimum bound of group $V_{i}$ is the largest number of matched pairs from group $V_{i}$ if only exchanges between vertices within group $V_{i}$ are allowed. The modified minimum bound is critical to define individual rationality.

\section{Desirable Properties}

For our problem, an algorithm takes as input a compatibility graph $G$, and outputs a matching $M$ as the outcome. Next we introduce three desirable properties that an outcome should satisfy. The first property is optimality, which requires that a matching must maximize the number of exchanges among compatible patient-donor pairs.

Definition 1 (Optimality). Given a compatible graph $G, a$ matching $M$ in $G$ satisfies optimality if $M$ is a maximum matching in $G$.

The second property, individual rationality, requires that in an individually rational matching $M$, the number of matched pairs $\left|M_{i}\right|$ of each group $V_{i}$ not be less than its modified minimum bound $\widetilde{\min }\left(V_{i}\right)$. This property guarantees that each group $V_{i}$ will receive at least the same number of matched pairs as that when group $V_{i}$ conducts exchanges by itself.

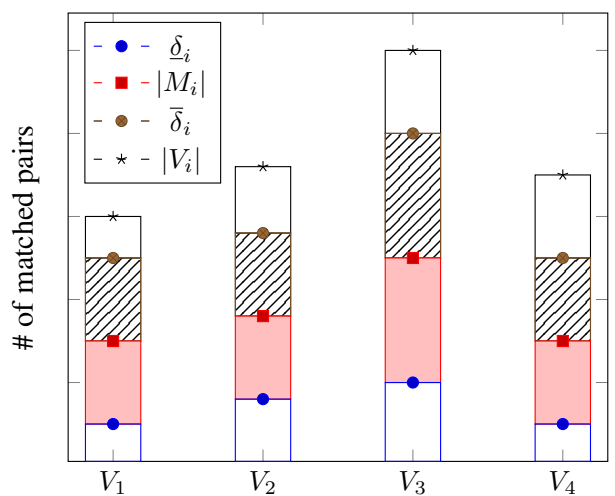

Figure 1: Given a matching $M$, for each group $V_{i}$, colored region represents $\left|M_{i}\right|-\underline{\delta}_{i}$ and shaded region represents $\bar{\delta}_{i}-\left|M_{i}\right|$. Height of each bar equals size $\left|V_{i}\right|$ of each group.

Definition 2 (Individual Rationality). Given a compatibility graph $G=(V, E)$ and a partition $P$ of vertices $V$, a matching $M$ in $G$ satisfies individual rationality, if for each group $V_{i} \in P$, we have $\left|M_{i}\right| \geq \widetilde{\min }\left(V_{i}\right)$.

Note that for compatible graphs with identical neighbors, individual rationality is trivially satisfied, because each group forms an independent set and the modified minimum bound is zero for each group.

Next we introduce an important notion called selection ratio which is key to the definition of our third property fairness. Let $\bar{\delta}_{i}$ and $\underline{\delta}_{i}$ denote the upper and lower bounds for group $V_{i}$ with $\bar{\delta}_{i} \geq \underline{\delta}_{i}$. Intuitively, these two bounds represent two target quotas s.t. we expect the number of matched pairs from group $V_{i}$ to fall within the range of these two bounds.

Given a matching $M$, upper and lower bounds $\bar{\delta}_{i}$ and $\underline{\delta}_{i}$, selection ratio $\alpha\left(\left|M_{i}\right|, \bar{\delta}_{i}, \underline{\delta}_{i}\right)$ of group $V_{i}$ is represented as a fraction, where the numerator is the difference between the number of matched pairs $\left|M_{i}\right|$ from $V_{i}$ in matching $M$ and the lower bound $\underline{\delta}_{i}$, and the denominator is the difference between upper bound $\bar{\delta}_{i}$ and lower bound $\underline{\delta}_{i}$. Formally,

Definition 3 (Selection Ratio). Given a matching $M$ in $G$, two quotas $\bar{\delta}_{i}$ and $\underline{\delta}_{i}$ with $\bar{\delta}_{i} \geq \underline{\delta}_{i}$, selection ratio of group $V_{i}$ w.r.t. $\bar{\delta}_{i}$ and $\underline{\delta}_{i}$ is

$$
\alpha\left(\left|M_{i}\right|, \bar{\delta}_{i}, \underline{\delta}_{i}\right)=\frac{\left|M_{i}\right|-\underline{\delta}_{i}}{\bar{\delta}_{i}-\underline{\delta}_{i}} \quad \text { for } \bar{\delta}_{i}>\underline{\delta}_{i}
$$

When $\bar{\delta}_{i}=\underline{\delta}_{i}$, we assume $\alpha\left(\left|M_{i}\right|, \bar{\delta}_{i}, \underline{\delta}_{i}\right)=-\infty$ if $\left|M_{i}\right|<\bar{\delta}_{i}$ and $\alpha\left(\left|M_{i}\right|, \bar{\delta}_{i}, \underline{\delta}_{i}\right)=\infty$ if $\left|M_{i}\right| \geq \bar{\delta}_{i}$.

Selection ratio measures to what extent the number of matched pairs $\left|M_{i}\right|$ from group $V_{i}$ surpasses lower bound $\underline{\delta}_{i}$ on the scale of the lower bound to the upper bound, as shown in Figure 1. Note that it is flexible to choose upper and lower bounds in the formula of a selection ratio, which allows us to capture different reasonable ideas, e.g., egalitarianism and proportionality. We discuss different choices of upper and lower bounds in Section 4.

The third property, fairness among groups, requires that the minimum selection ratio among all the groups be maximized 
among all matchings. This is a natural and unified fairness concept for different compatibility graph structures.

Definition 4 (Fairness among Groups). Given the set of all matchings $\mathbb{M}$ in $G$, a partition $P$ of vertices $V$ and two vectors of quotas $\bar{\delta}=\left(\bar{\delta}_{i}\right)_{V_{i} \in P}$ and $\underline{\delta}=\left(\underline{\delta}_{i}\right)_{V_{i} \in P}$ with $\bar{\delta}_{i} \geq \underline{\delta}_{i}$ for each group $V_{i} \in P$, a matching $M$ in $G$ is fair (among groups) w.r.t. $\bar{\delta}$ and $\underline{\delta}$ if it maximizes the minimal selection ratio among all groups:

$$
M \in \underset{M^{\prime} \in \mathbb{M}}{\arg \max } \min _{V_{i} \in P} \alpha\left(\left|M_{i}^{\prime}\right|, \bar{\delta}_{i}, \underline{\delta}_{i}\right)
$$

W.L.O.G, we assume that $\bar{\delta}_{i} \geq \underline{\delta}_{i}$ for each group $V_{i} \in P$ for the rest of the paper.

\section{Choice of Upper and Lower Bounds}

In this section, we propose several choices of upper and lower bounds and assign different names to the corresponding selection ratios summarized in Table 1.

\subsection{Egalitarianism}

For each group $V_{i}$, if we set $\bar{\delta}_{i}=|V|$ and $\underline{\delta}_{i}=0$, then the egalitarian-selection-ratio (Egalitarian) $\alpha(\cdot)$ represents the ratio between the number of matched pairs $\left|M_{i}\right|$ from group $V_{i}$ and the total number of vertices $|V|$.

$$
\alpha\left(\left|M_{i}\right|, \bar{\delta}_{i}, \underline{\delta}_{i}\right)=\frac{\left|M_{i}\right|-\underline{\delta}_{i}}{\bar{\delta}_{i}-\underline{\delta}_{i}}=\frac{\left|M_{i}\right|}{|V|}
$$

A fair matching w.r.t. Egalitarian tries to equalize the numbers of matched pairs among all groups. Note that another positive constant can be chosen as $\bar{\delta}_{i}$ instead of $|V|$.

\subsection{Proportional to Group Sizes (Group-Size)}

For each group $V_{i}$, if we set $\bar{\delta}_{i}=\left|V_{i}\right|$ and $\underline{\delta}_{i}=0$, then the group-size-selection-ratio (Group-Size) $\alpha(\cdot)$ represents the ratio between the number of matched pairs $\left|M_{i}\right|$ from group $V_{i}$ and its group size $\left|V_{i}\right|$.

$$
\alpha\left(\left|M_{i}\right|, \bar{\delta}_{i}, \underline{\delta}_{i}\right)=\frac{\left|M_{i}\right|-\underline{\delta}_{i}}{\bar{\delta}_{i}-\underline{\delta}_{i}}=\frac{\left|M_{i}\right|}{\left|V_{i}\right|}
$$

A fair matching w.r.t. Group-Size tries to ensure that for each group, the number of matched pairs $\left|M_{i}\right|$ from group $V_{i}$ is proportional to its group size $\left|V_{i}\right|$.

\subsection{Proportional to Maximum Bounds (Maximum)}

For each group $V_{i}$, if we set $\bar{\delta}_{i}=\max \left(V_{i}\right)$ and $\underline{\delta}_{i}=0$, then the maximum-bound-selection-ratio (Maximum) $\alpha(\cdot)$ represents the ratio between the number of matched pairs $\left|M_{i}\right|$ from group $V_{i}$ and its maximum bound $\max \left(V_{i}\right)$.

$$
\alpha\left(\left|M_{i}\right|, \bar{\delta}_{i}, \underline{\delta}_{i}\right)=\frac{\left|M_{i}\right|-\underline{\delta}_{i}}{\bar{\delta}_{i}-\underline{\delta}_{i}}=\frac{\left|M_{i}\right|}{\max \left(V_{i}\right)}
$$

A fair matching w.r.t. Maximum tries to ensure that for each group, the number of matched pairs $\left|M_{i}\right|$ from group $V_{i}$ is proportional to its maximum bound $\max \left(V_{i}\right)$.

\begin{tabular}{|c|c|c|}
\hline & Upper bound & Lower bound \\
\hline Egalitarian & $|V|$ & 0 \\
\hline Group-Size & $\left|V_{i}\right|$ & 0 \\
\hline Maximum & $\max \left(V_{i}\right)$ & 0 \\
\hline Minimum & $\min \left(V_{i}\right)+1$ & 0 \\
\hline Max-Min & $\max \left(V_{i}\right)$ & $\min \left(V_{i}\right)$ \\
\hline Max-M-Min & $\max \left(V_{i}\right)$ & $\widehat{\min }\left(V_{i}\right)$ \\
\hline
\end{tabular}

Table 1: Different Choices of Upper and Lower Bounds

\subsection{Proportional to Minimum Bounds (Minimum)}

For each group $V_{i}$, if we set $\bar{\delta}_{i}=\min \left(V_{i}\right)+1$ and $\underline{\delta}_{i}=0$, then the minimum-bound-selection-ratio (Minimum) $\alpha(\cdot)$ represents the ratio between the number of matched pairs $\left|M_{i}\right|$ from group $V_{i}$ and its minimum bound $\min \left(V_{i}\right)$ plus 1.

$$
\alpha\left(\left|M_{i}\right|, \bar{\delta}_{i}, \underline{\delta}_{i}\right)=\frac{\left|M_{i}\right|-\underline{\delta}_{i}}{\bar{\delta}_{i}-\underline{\delta}_{i}}=\frac{\left|M_{i}\right|}{\min \left(V_{i}\right)+1}
$$

A fair matching w.r.t. Minimum tries to ensure that for each group, the number of matched pairs $\left|M_{i}\right|$ from group $V_{i}$ is proportional to its minimum bound $\min \left(V_{i}\right)$ plus 1 . Note that we consider minimum bound $\min \left(V_{i}\right)$ plus 1 to avoid the case that $\min \left(V_{i}\right)=0$. Similarly, we can replace minimum bound $\min \left(V_{i}\right)$ by modified minimum bound $\widetilde{\min }\left(V_{i}\right)$.

\subsection{Proportional to the Range of Maximum and Minimum Bounds (Max-Min)}

For each group $V_{i}$, if we set $\bar{\delta}_{i}=\max \left(V_{i}\right)$ and $\underline{\delta}_{i}=\min \left(V_{i}\right)$, then the maximum-minimum-selection-ratio (Max-Min) $\alpha(\cdot)$ represents the ratio between the number of matched pairs $\left|M_{i}\right|$ from group $V_{i}$ minus its minimum bound and the difference between its maximum and minimum bounds.

$$
\alpha\left(\left|M_{i}\right|, \bar{\delta}_{i}, \underline{\delta}_{i}\right)=\frac{\left|M_{i}\right|-\underline{\delta}_{i}}{\bar{\delta}_{i}-\underline{\delta}_{i}}=\frac{\left|M_{i}\right|-\min \left(V_{i}\right)}{\max \left(V_{i}\right)-\min \left(V_{i}\right)}
$$

A fair matching w.r.t. Max-Min tries to ensure that for each group $V_{i}$, the number of matched pairs $\left|M_{i}\right|$ from group $V_{i}$ minus its minimum bound is proportional to the difference between its maximum and minimum bounds.

\subsection{Proportional to the Range of Maximum and Modified Minimum Bounds (Max-M-Min)}

For each group $V_{i}$, if we set $\bar{\delta}_{i}=\max \left(V_{i}\right)$ and $\underline{\delta}_{i}=\widetilde{\min }\left(V_{i}\right)$, then the maximum-modified-minimum-selection-ratio (MaxM-Min) $\alpha(\cdot)$ represents the ratio between the number of matched pairs $\left|M_{i}\right|$ from group $V_{i}$ minus its minimum bound and the difference between its maximum and modified minimum bounds.

$$
\alpha\left(\left|M_{i}\right|, \bar{\delta}_{i}, \underline{\delta}_{i}\right)=\frac{\left|M_{i}\right|-\underline{\delta}_{i}}{\bar{\delta}_{i}-\underline{\delta}_{i}}=\frac{\left|M_{i}\right|-\widetilde{\min }\left(V_{i}\right)}{\max \left(V_{i}\right)-\widetilde{\min }\left(V_{i}\right)}
$$

A fair matching w.r.t. Max-M-Min tries to ensure that for each group, the number of matched pairs $\left|M_{i}\right|$ from group $V_{i}$ minus its modified minimum bound is proportional to the difference between its maximum and modified minimum bounds. 


\section{Compatibility between Optimality, Individual Rationality and Fairness}

In this section, we discuss whether a matching always exists that satisfies optimality, individual rationality and fairness w.r.t. the different choices of upper and lower bounds in Table 1. The results are different for compatibility graphs with identical and different neighbors, as summarized in Theorem 1 and Theorem 2.

Theorem 1. For compatibility graphs with identical neighbors, a matching always exists that satisfies optimality, individual rationality, and fairness w.r.t. any choice of upper and lower bounds in Table 1.

Proof. Given a compatibility graph $G$ with identical neighbors, let $M^{\prime}$ denote any fair matching w.r.t. any choice of upper and lower bounds in Table 1 . If $M^{\prime}$ is not a maximum matching in $G$, by Berge's theorem [Berge, 1957], then an augmenting path $p$ must exist w.r.t. $M^{\prime}$ such that we can update $M^{\prime}$ to be a new matching $M^{\prime} \oplus p$ in which the edges from $p \backslash M^{\prime}$ are added while those from $M^{\prime} \cap p$ are removed. Note that the vertices that are matched in $M^{\prime}$ remain matched in new matching $M^{\prime} \oplus p$. Eventually we can obtain a maximum matching $M$ by iteratively finding all augmenting paths w.r.t. $M^{\prime}$, and for each group $V_{i}$, the number of matched pairs $\left|M_{i}\right|$ in $M$ is at least as large as the number of matched pairs $\left|M_{i}^{\prime}\right|$ in $M^{\prime}$. Then the minimum selection ratio in matching $M$ remains the same as in $M^{\prime}$; otherwise $M^{\prime}$ cannot be a fair matching. By the definition of fairness, the maximum matching $M$ is also a fair matching.

Next we show that matching $M$ also satisfies individual rationality. Recall that we assume all patient-donor pairs in each vertex are incompatible, which indicates that there is no edge between two vertices from the same group. Thus for each group $V_{i}$, its modified minimum bound $\widetilde{\min }\left(V_{i}\right)$ is always 0 . Then for any maximum matching $M^{\prime}$ and for each group $V_{i}, \min \left(V_{i}\right) \leq\left|M_{i}\right| \leq \max \left(V_{i}\right)$ holds. Since $\widetilde{\min }\left(V_{i}\right)$ $=0 \leq \min \left(V_{i}\right)$, then any maximum matching in $G$ is also individually rational. This completes the proof.

Theorem 2. For compatibility graphs with different neighbors, a matching always exists that satisfies optimality, individual rationality and fairness w.r.t. Max-M-Min. Fairness w.r.t. any other choice of upper and lower bounds in Table 1 is incompatible with optimality and individual rationality.

Proof. Next we show that given a compatibility graph $G$ with different neighbors, fairness w.r.t. Max-M-Min is compatible with optimality and individual rationality. Let $M$ denote any fair matching w.r.t. Max-M-Min in $G$. If $M$ is not a maximum matching, then we can update $M$ to be a maximum matching as discussed in the proof for Theorem 1. For the sake of contradiction, suppose $M$ does not satisfy individual rationality. Then there must exist a group $V_{i}$ such that $\left|M_{i}\right|$ $<\widetilde{\min }\left(V_{i}\right)$ and therefore its selection ratio $\alpha(\cdot)$ is negative (including where $\widetilde{\min }\left(V_{i}\right)=\max \left(V_{i}\right)$ ).

Now consider another matching $M^{*}$ obtained as follows. For each group $V_{i}$, let $O_{i}$ denote a maximum matching in subgraph $G\left[V_{i}\right]$ only induced by vertices from group $V_{i}$. Match- ing $M^{*}=\bigcup_{V_{i} \in P} O_{i}$ is the combination of each maximum matching $O_{i}$ in each corresponding subgraph $G\left[V_{i}\right]$. Then for each group, $\left|M_{i}^{*}\right|=\widetilde{\min }\left(V_{i}\right)$ holds, which satisfies individual rationality. However, this leads to a contradiction where matching $M$ maximizes the minimal selection ratio among all the groups. Thus the assumption is wrong, and a maximum and fair matching w.r.t. Max-M-Min also satisfies individual rationality.

We prove the second part of Theorem 2 by a counterexample in the Appendix due to space limitation.

\section{Algorithm Design}

In this section, we present a general algorithm that computes a matching that achieves optimality, individual rationality and fairness w.r.t. the different choices of upper and lower bounds in Table 1. The algorithm works for compatibility graphs with both identical and different neighbors and yields either a matching satisfying three properties whenever one exists or a NO-instance otherwise, as described in Algorithm 2.

\subsection{Computing a Fair Matching}

Next we describe the first step of Algorithm 2 that computes a fair matching w.r.t. some choice of upper and lower quotas in Table 1, as described in Algorithm 1.

To develop a good intuition of how Algorithm 1 works, we postpone the following two technical details later: i) Some choices of upper and lower bounds require the maximum and minimum bounds of all groups. ii) Algorithm 1 iteratively invokes Algorithm $\Gamma$ that solves the following problem of Matching with Quotas. Intuitively, Algorithm $\Gamma$ checks whether a matching $M$ exists s.t. for each group $V_{i}$, the number of matched pairs $\left|M_{i}\right|$ is not smaller than some target quota $\lambda_{i}$. We design efficient algorithms regarding these two issues in Section 7.

\begin{tabular}{|ll|}
\hline Matching with Quotas \\
Input: & A compatibility graph $G$, a partition $P$ of \\
& vertices $V$, a vector of targets $\lambda=\left(\lambda_{i}\right)_{V_{i} \in P .}$ \\
Question: & $\begin{array}{l}\text { Whether a matching } M \text { in } G \text { exists s.t. for } \\
\text { each } V_{i} \in P, \lambda_{i} \leq\left|M_{i}\right| \text { holds. }\end{array}$ \\
\hline
\end{tabular}

The basic idea of Algorithm 1 is to apply a binary search to find the maximal selection ratio $\alpha$ such that a matching exists where each group has a weakly larger selection ratio than $\alpha$. Algorithm 1 takes as input a compatibility graph $G$, a vector of upper bounds $\bar{\delta}=\left(\bar{\delta}_{i}\right)_{1 \ldots k}$ and a vector of lower bounds $\underline{\delta}=\left(\underline{\delta}_{i}\right)_{1 \ldots k}$. During the process of Algorithm 1, we track three variables, $\alpha, \alpha_{\ell}$, and $\alpha_{u}$, representing the current, the lower, and the upper selection ratios respectively. In the initialization step, we set lower selection ratio $\alpha_{\ell}$ to 0 and set upper selection ratio $\alpha_{u}$ to 1 . For each group $V_{i}$, we initialize its target quota $\lambda_{i}$ to 0 . During each round of Algorithm 1, first, compute current selection ratio $\alpha=\left(\alpha_{\ell}+\alpha_{u}\right) / 2$. Then for each group $V_{i}$, calculate its target quota $\lambda_{i}$ w.r.t. $\alpha$, by rounding up value $\left\lceil\alpha \cdot\left(\bar{\delta}_{i}-\underline{\delta}_{i}\right)+\underline{\delta}_{i}\right\rceil$. Then we check whether a matching $M$ exists s.t. for each group $V_{i},\left|M_{i}\right| \geq \lambda_{i}$ holds through Algorithm $\Gamma$. If so, then update lower selection ratio $\alpha_{\ell}$ to be $\alpha$ to search for a larger selection ratio in the range 


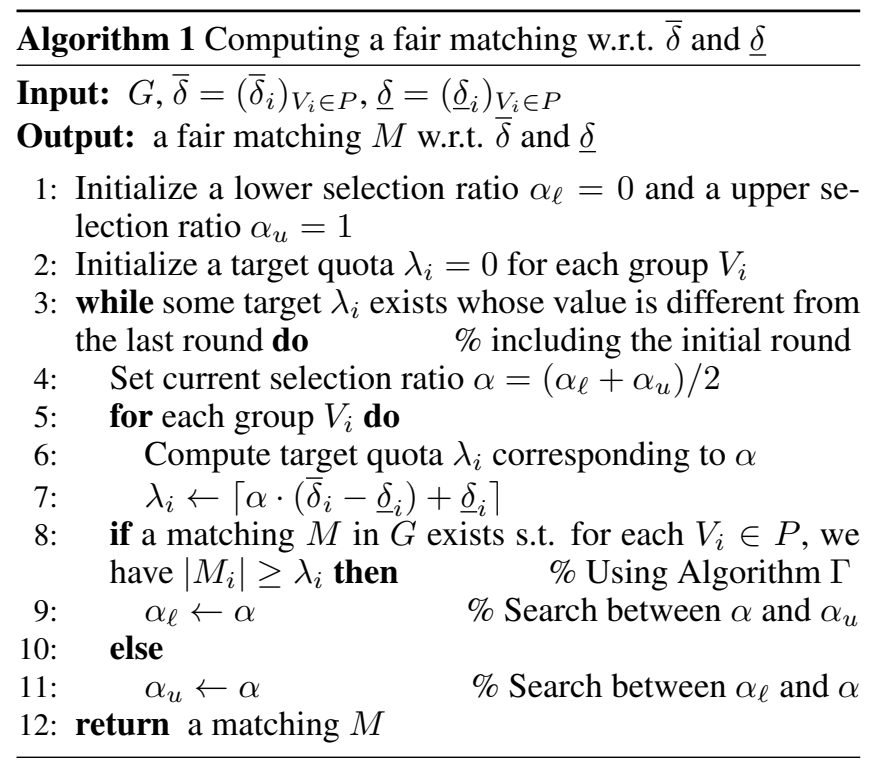

$\left[\alpha, \alpha_{u}\right]$ in the next round; otherwise, update upper selection ratio $\alpha_{u}$ to be $\alpha$ to search for a smaller selection ratio in the range $\left[\alpha_{\ell}, \alpha\right]$ in the next round. Repeat these procedures whenever some target $\lambda_{i}$ exists whose value is different from the last round; otherwise Algorithm 2 terminates.

Theorem 3. Given a compatibility graph $G$ and a choice of upper and lower bounds in Table 1, Algorithm 1 yields a fair matching in polynomial time.

\subsection{Computing a Maximum, Individually Rational and Fair Matching}

Next we give a high-level description of Algorithm 2 that verifies the existence of a matching that achieves optimality, individual rationality and fairness w.r.t. some choice of upper and lower bounds in Table 1.

The input consists of a compatibility graph $G$ and two vectors of quotas $\bar{\delta}$ and $\underline{\delta}$. The first step computes a fair matching $M^{\prime}$ through Algorithm 1. We use $\alpha$ to denote the minimal selection ratio among all groups. The second step employs Algorithm $\Gamma$ to check whether a fair and individually rational matching $M$ exists s.t. for each group $V_{i}$, we have $\max \left(\left\lceil\alpha \cdot\left(\bar{\delta}_{i}-\underline{\delta}_{i}\right)+\underline{\delta}_{i}\right\rceil, \widetilde{\min }\left(V_{i}\right)\right) \leq\left|M_{i}\right|$. In the final step, if a fair and individually rational matching $M$ exists, then we update $M$ to be a maximum matching. Otherwise, no such matching exists.

Theorem 4. Given a compatibility graph and some choice of upper and lower bounds in Table 1, Algorithm 2 finds a matching that satisfies optimality, individual rationality and fairness whenever it exists in polynomial time.

\section{Running Time Upper Bounds}

This section is devoted to the two remaining technical details in Section 6: i) how to compute the maximum and minimum bounds for each group and ii) how to design Algorithm $\Gamma$. We present different polynomial-time algorithms for different compatibility graph structures and summarize all the results
Algorithm 2 Checking existence of a matching satisfying optimality, individual rationality, and fairness w.r.t. $\bar{\delta}$ and $\underline{\delta}$

Input: $G, \bar{\delta}=\left(\bar{\delta}_{i}\right)_{V_{i} \in P}, \underline{\delta}=\left(\underline{\delta}_{i}\right)_{V_{i} \in P}$

Output: a matching $M$ that is maximum, individually rational and fair w.r.t. $\bar{\delta}$ and $\underline{\delta}$

1: Compute a fair matching $M^{\prime}$ in $G$ w.r.t. $\bar{\delta}$ and $\underline{\delta}$ and let $\alpha$ denote the minimal selection ratio among all groups $\%$ using Algorithm 1

2: if a matching $M$ exists s.t. for each group $V_{i}$, we have $\max \left(\left\lceil\alpha \cdot\left(\bar{\delta}_{i}-\underline{\delta}_{i}\right)+\underline{\delta}_{i}\right\rceil, \widetilde{\min }\left(V_{i}\right)\right) \leq\left|M_{i}\right| \quad \%$ using Algorithm $\Gamma$ then

3: $\quad$ Update $M$ to be a maximum matching.

4: $\quad$ return matching $M$

5: else

6: return $\mathrm{NO}$-instance

on running time upper bounds in Table 2. Detailed proofs are presented in the Appendix.

Note that given a compatible graph with identical neighbors $G=(V, E)$ with a partition $P$ of vertices $V$, we can create an equivalent and compact graph $G^{\prime}=\left(V^{\prime}, E^{\prime}\right)$ in which each node $V_{i} \in V^{\prime}$ represents a group with capacity $b\left(V_{i}\right)=\left|V_{i}\right|$, i.e., the size of group $V_{i}$. We can construct such a compact representation graph $G^{\prime}$ in polynomial time and assume the input for any compatibility graph with identical neighbors is its compact representation. The details of constructing compact graphs are presented in the Appendix.

\subsection{Bipartite Graphs with Identical Neighbors}

First, we consider the simplest model, bipartite compatibility graphs with identical neighbors in which all groups form a bipartite graph and all vertices within each group have identical neighbors [Ergin et al., 2017].

Theorem 5. Given a bipartite compatibility graph with identical neighbors, the maximum bounds of all groups can be computed in time $O\left(k^{2}\right)$ where $k$ is the number of groups.

Proof. (Sketch) Let $N_{G}\left(V_{i}\right)$ denote all neighboring vertices of some vertex from group $V_{i}$ in $G$. For each group $V_{i}$, its maximum bound $\max \left(V_{i}\right)$ equals $\min \left(\left|V_{i}\right|,\left|N_{G}\left(V_{i}\right)\right|\right)$ and we can calculate the total number of its neighboring vertices in $O(k)$. Thus the total running time is $O\left(k^{2}\right)$.

Theorem 6. Given a bipartite compatibility graph with identical neighbors, the minimum bounds of all groups can be computed in time $O\left(k^{3.5}\right)$ where $k$ is the number of groups.

\begin{tabular}{l|c|c|c}
\hline & $\begin{array}{c}\text { Identical } \\
\text { Bipartite }\end{array}$ & $\begin{array}{c}\text { Identical } \\
\text { Non-bipartite }\end{array}$ & $\begin{array}{c}\text { Different } \\
\text { Non-bipartite }\end{array}$ \\
\hline Maximum & $O\left(k^{2}\right)$ & $O\left(k^{2}\right)$ & $O(|V| \cdot|E|)$ \\
\hline Minimum & $O\left(k^{3.5}\right)$ & $O(|V| \cdot|E|)$ & $O(|V| \cdot|E|)$ \\
\hline Algorithm $\Gamma$ & $O\left(k^{3}\right)$ & $O\left(k^{4} \cdot \log k\right)$ & $O(\sqrt{|V|} \cdot|E|)$ \\
\hline
\end{tabular}

Table 2: Running time upper bounds where $k,|V|$, and $|E|$ represent numbers of groups, vertices, and edges in $G$ 
Proof. (Sketch) Let $M$ denote a maximum matching in $G$ and let $M^{\prime}$ denote a maximum matching in subgraph $G\left[V \backslash V_{i}\right]$ induced from all groups excluding group $V_{i}$. For each group $V_{i}$, its minimum bound $\min \left(V_{i}\right)$ is $|M|-\left|M^{\prime}\right|$. Note that we can compute a maximum matching in bipartite compact graph $G^{\prime}$ of $G$ by the Hopcroft-Karp algorithm in time $O\left(k^{2.5}\right)$ [Hopcroft and Karp, 1973]. Thus the total running time for computing all minimum bounds is $O\left(k^{3.5}\right)$.

Theorem 7. Given a bipartite compatibility graph $G$ with identical neighbors and a vector of quotas $\lambda=\left(\lambda_{i}\right)_{V_{i} \in P}$ where each element $\lambda_{i}$ corresponds to one group $V_{i}$, checking whether a matching $M$ in $G$ exists s.t. $\lambda_{i} \leq\left|M_{i}\right|$ holds for each group $V_{i}$ can be done in time $O\left(k^{3}\right)$ where $k$ is the number of groups.

Proof. (Sketch) We prove Theorem 7 by converting the problem of checking whether a matching $M$ in $G$ exists s.t. for each group $V_{i}, \lambda_{i} \leq\left|M_{i}\right|$ holds into an equivalent network flow problem with edge capacities in polynomial time, which then can be solved in time $O\left(k^{3}\right)$ [Malhotra et al., 1978].

\subsection{Non-bipartite Graphs with Identical Neighbors}

Next we consider non-bipartite compatibility graphs with identical neighbors in which all vertices within each group have identical neighbors [Dickerson et al., 2017].

Theorem 8. Given a non-bipartite compatibility graph with identical neighbors, the maximum bounds of all groups can be computed in time $O\left(k^{2}\right)$ where $k$ is the number of groups.

Proof. (Sketch) The same proof for Theorem 5 works.

Theorem 9. Given a non-bipartite compatibility graph $G=$ $(V, E)$ with identical neighbors, the minimum bounds of all groups can be computed in time $O(|V| \cdot|E|)$ where $|V|$ and $|E|$ denote the numbers of vertices and edges in $G$.

Proof. (Sketch) Consider any maximum matching $M$ in $G$. If an alternating path $p$ exists that starts from some unmatched vertex $u \in V \backslash V_{i}$ and ends at some matched vertex $v \in$ $V_{i}$ w.r.t. $M$, then update $M$ to be $M \oplus p$ by taking their symmetric difference. Repeat this procedure until there is no such alternating path w.r.t. $M$, and the minimum bound of group $V_{i}$ is $\left|M_{i}\right|$. For each group $V_{i}$, we can compute its minimum bound in time $O\left(\left|V_{i}\right| \cdot|E|\right)$. The total running time of computing all minimum bounds is $O(|V| \cdot|E|)$.

Theorem 10. Given a non-bipartite compatibility graph $G$ with identical neighbors and a vector of quotas $\lambda=\left(\lambda_{i}\right)_{V_{i} \in P}$ where each element $\lambda_{i}$ corresponds to one group $V_{i}$, checking whether a matching $M$ in $G$ exists s.t. $\lambda_{i} \leq\left|M_{i}\right|$ holds for each group $V_{i}$ can be done in time $O\left(k^{4} \cdot \log k\right)$ where $k$ is the number of groups.

Proof. (Sketch) We prove Theorem 10 by converting the problem of checking whether a matching $M$ in $G$ exists s.t. for each group $V_{i}, \lambda_{i} \leq\left|M_{i}\right|$ holds into an equivalent bmatching problem in polynomial time, which then can be solved in time $O\left(k^{4} \cdot \log k\right)$ [Anstee, 1987].

\subsection{Compatible Graphs with Different Neighbors}

Next we consider compatibility graphs with different neighbors where vertices from the same group may have different neighbors [Mattei et al., 2017; Biró et al., 2019].

Theorem 11. Given a compatibility graph $G=(V, E)$ with different neighbors, the maximum bounds of all groups can be computed in time $O(|V| \cdot|E|)$.

Proof. (Sketch) We use almost the same proof as that for Theorem 9. The main difference is that for computing the maximum bound, we continue to seek an alternating path $p$ that starts from some unmatched vertex $v \in V_{i}$ and ends at some matched vertex $u \in V \backslash V_{i}$ w.r.t. $M$. Thus they have the same running time $O(|V| \cdot|E|)$.

Theorem 12. Given a compatibility graph $G=(V, E)$ with different neighbors, the minimum bounds of all groups can be computed in time $O(|V| \cdot|E|)$.

Proof. (Sketch) The same proof for Theorem 9 works.

Theorem 13. Given a compatibility graph $G$ with different neighbors and a vector of quotas $\lambda=\left(\lambda_{i}\right)_{V_{i} \in P}$ where each element $\lambda_{i}$ corresponds to one group $V_{i}$, checking whether a matching $M$ in $G$ exists s.t. $\lambda_{i} \leq\left|M_{i}\right|$ holds for each group $V_{i}$ can be done in time $O(\sqrt{|V|} \cdot|E|)$.

Proof. (Sketch) Create a new graph $G^{*}$ by extending $G=$ $(V, E)$ as follows. For each group $V_{i}$, add a new set of $\left|V_{i}\right|-$ $\lambda_{i}$ vertices, denoted by $V_{i}^{\prime}$. Each newly added vertex $v_{i}^{\prime} \in$ $V_{i}^{\prime}$ is incident to all vertices of $V_{i}$. All newly added vertices $\bigcup_{V_{i} \in P} V_{i}^{\prime}$ are incident to each other. If the total number of vertices is odd, then add one more vertex $v^{*}$ that is incident to all newly added vertices $\bigcup_{V_{i} \in P} V_{i}^{\prime}$. There exists a matching $M$ in $G$ s.t. $\lambda_{i} \leq\left|M_{i}\right|$ holds for each group $V_{i}$ if and only if induced graph $G^{*}$ has a perfect matching $M^{*}$. We can check whether $G^{*}$ admits a perfect matching by computing a maximum matching in time $O\left(\sqrt{\left|V^{*}\right|} \cdot\left|E^{*}\right|\right)$ [Micali and Vazirani, 1980] where $\left|V^{*}\right|$ and $\left|E^{*}\right|$ are linear in the numbers of $|V|$ and $|E|$.

\section{Conclusion}

We studied the pairwise organ exchange problem among groups motivated by real-world organ allocation markets. We proposed a new and straightforward fairness concept and introduced a general polynomial-time algorithm that computes a matching that satisfies three desirable properties including optimality, individual rationality, and fairness. An interesting future direction is how to design a fair and efficient algorithm for exchange among groups by relaxing the assumption of pairwise exchanges.

\section{Acknowledgements}

The authors appreciate Haris Aziz and Serge Gaspers for their constructive comments. This work is partially supported by JSPS KAKENHI Grant Numbers JP20H00587. Toby Walsh is supported by the Australian Research Council via a Laureate Fellowship FL200100204. 


\section{References}

[Abraham et al., 2007] D. J. Abraham, A. Blum, and T. Sandholm. Clearing algorithms for barter exchange markets: Enabling nationwide kidney exchanges. In Proceedings of the 8th ACM conference on Electronic commerce, pages 295-304, 2007.

[Anstee, 1987] R. P. Anstee. A polynomial algorithm for bmatchings: an alternative approach. Information Processing Letters, 24(3):153-157, 1987.

[Ashlagi et al., 2015] I. Ashlagi, F. Fischer, I. A. Kash, and A. D. Procaccia. Mix and match: A strategyproof mechanism for multi-hospital kidney exchange. Games and Economic Behavior, 91:284-296, 2015.

[Berge, 1957] C. Berge. Two theorems in graph theory. Proceedings of the National Academy of Sciences of the United States of America, 43(9):842, 1957.

[Bertsimas et al., 2013] D. Bertsimas, V. F. Farias, and N. Trichakis. Fairness, efficiency, and flexibility in organ allocation for kidney transplantation. Operations Research, 61(1):73-87, 2013.

[Biró et al., 2019] P. Biró, W. Kern, D. Pálvölgyi, and D. Paulusma. Generalized matching games for international kidney exchange. In Proceedings of the 18th International Conference on Autonomous Agents and Multiagent Systems (AAMAS), pages 413-421, 2019.

[Dickerson and Sandholm, 2017] J. P. Dickerson and T. Sandholm. Multi-organ exchange. Journal of Artificial Intelligence Research, 60:639-679, 2017.

[Dickerson et al., 2014] J. P. Dickerson, A. D. Procaccia, and T. Sandholm. Price of fairness in kidney exchange. Transplantation, 98:815, 2014.

[Dickerson et al., 2017] J. P. Dickerson, A. M. Kazachkov, A. D. Procaccia, and T. Sandholm. Small representations of big kidney exchange graphs. In Proceedings of the 31st International Joint Conference on Artificial Intelligence (IJCAI), pages 487-493, 2017.

[Dickerson et al., 2019] J. P. Dickerson, A. D. Procaccia, and T. Sandholm. Failure-aware kidney exchange. Management Science, 65(4):1768-1791, 2019.

[Ergin et al., 2017] H. Ergin, T. Sönmez, and M. U. Ünver. Dual-donor organ exchange. Econometrica, 85(5):16451671, 2017.

[Ergin et al., 2020] H. Ergin, T. Sönmez, and M. U. Ünver. Efficient and incentive-compatible liver exchange. Econometrica, 88(3):965-1005, 2020.

[Freedman et al., 2020] R. B. Freedman, S. Jana, W. SinnottArmstrong, J. P. Dickerson, and V. Conitzer. Adapting a kidney exchange algorithm to align with human values. Artificial Intelligence, page 103261, 2020.

[Hajaj et al., 2015] C. Hajaj, J. P. Dickerson, A. Hassidim, T. Sandholm, and D. Sarne. Strategy-proof and efficient kidney exchange using a credit mechanism. In TwentyNinth AAAI conference on artificial intelligence, pages 921-928, 2015.
[Hopcroft and Karp, 1973] J. E. Hopcroft and R. M. Karp. An $n^{5 / 2}$ algorithm for maximum matchings in bipartite graphs. SIAM Journal on computing, 2(4):225-231, 1973.

[Malhotra et al., 1978] V. M. Malhotra, M. P. Kumar, and S. N. Maheshwari. An $O\left(|V|^{3}\right)$ algorithm for finding maximum flows in networks. Information Processing Letters, 7(6):277-278, 1978.

[Mattei et al., 2017] N. Mattei, A. Saffidine, and T. Walsh. Mechanisms for online organ matching. In Carles Sierra, editor, Proceedings of the 29st International Joint Conference on Artificial Intelligence (IJCAI), pages 345-351, 2017.

[Micali and Vazirani, 1980] S. Micali and V. V. Vazirani. An $O(\sqrt{|V|}|E|)$ algoithm for finding maximum matching in general graphs. In 21st Annual Symposium on Foundations of Computer Science (sfcs 1980), pages 17-27. IEEE, 1980.

[Roth et al., 2004] A. E. Roth, T. Sönmez, and M. U. Ünver. Kidney exchange. The Quarterly journal of economics, 119(2):457-488, 2004.

[Roth et al., 2005] A. E. Roth, T. Sönmez, and M. U. Ünver. Pairwise kidney exchange. Journal of Economic theory, 125(2):151-188, 2005.

[Roth et al., 2007] A. E. Roth, T. Sönmez, and M. U. Ünver. Efficient kidney exchange: Coincidence of wants in markets with compatibility-based preferences. American Economic Review, 97(3):828-851, 2007. 